\title{
Error-Driven Pruning of Treebank Grammars for Base Noun Phrase Identification
}

\author{
Claire Cardie and David Pierce \\ Department of Computer Science \\ Cornell University \\ Ithaca, NY 14853 \\ cardie, pierce@cs.cornell.edu
}

\begin{abstract}
Finding simple, non-recursive, base noun phrases is an important subtask for many natural language processing applications. While previous empirical methods for base NP identification have been rather complex, this paper instead proposes a very simple algorithm that is tailored to the relative simplicity of the task. In particular, we present a corpus-based approach for finding base NPs by matching part-ofspeech tag sequences. The training phase of the algorithm is based on two successful techniques: first the base NP grammar is read from a "treebank" corpus; then the grammar is improved by selecting rules with high "benefit" scores. Using this simple algorithm with a naive heuristic for matching rules, we achieve surprising accuracy in an evaluation on the Penn Treebank Wall Street Journal.
\end{abstract}

\section{Introduction}

Finding base noun phrases is a sensible first step for many natural language processing (NLP) tasks: Accurate identification of base noun phrases is arguably the most critical component of any partial parser; in addition, information retrieval systems rely on base noun phrases as the main source of multi-word indexing terms; furthermore, the psycholinguistic studies of Gee and Grosjean (1983) indicate that text chunks like base noun phrases play an important role in human language processing. In this work we define base NPs to be simple, nonrecursive noun phrases - noun phrases that do not contain other noun phrase descendants. The bracketed portions of Figure 1, for example, show the base NPs in one sentence from the Penn Treebank Wall Street Journal (WSJ) corpus (Marcus et al., 1993). Thus, the string the sunny confines of resort towns like Boca Raton and Hot Springs is too complex to be a base NP; instead, it contains four simpler noun phrases, each of which is considered a base NP: the sunny confines, resort towns, Boca Raton, and Hot Springs.

Previous empirical research has addressed the problem of base NP identification. Several algorithms identify "terminological phrases" - certain
When [it] is [time] for [their biannual powwow], [the nation] 's [manufacturing titans] typically jet off to [the sunny confines] of [resort towns] like [Boca Raton] and [Hot Springs].

Figure 1: Base NP Examples

base noun phrases with initial determiners and modifiers removed: Justeson \& Katz (1995) look for repeated phrases; Bourigault (1992) uses a handcrafted noun phrase grammar in conjunction with heuristics for finding maximal length noun phrases; Voutilainen's NPTool (1993) uses a handcrafted lexicon and constraint grammar to find terminological noun phrases that include phrase-final prepositional phrases. Church's PARTS program (1988), on the other hand, uses a probabilistic model automatically trained on the Brown corpus to locate core noun phrases as well as to assign parts of speech. More recently, Ramshaw \& Marcus (In press) apply transformation-based learning (Brill, 1995) to the problem. Unfortunately, it is difficult to directly compare approaches. Each method uses a slightly different definition of base NP. Each is evaluated on a different corpus. Most approaches have been evaluated by hand on a small test set rather than by automatic comparison to a large test corpus annotated by an impartial third party. A notable exception is the Ramshaw \& Marcus work, which evaluates their transformation-based learning approach on a base NP corpus derived from the Penn Treebank WSJ, and achieves precision and recall levels of approximately $93 \%$.

This paper presents a new algorithm for identifying base NPs in an arbitrary text. Like some of the earlier work on base NP identification, ours is a trainable, corpus-based algorithm. In contrast to other corpus-based approaches, however, we hypothesized that the relatively simple nature of base NPs would permit their accurate identification using correspondingly simple methods. Assume, for example, that we use the annotated text of Figure 1 as our training corpus. To identify base NPs in an unseen 
text, we could simply search for all occurrences of the base NPs seen during training - it, time, their biannual powwow, ..., Hot Springs - and mark them as base NPs in the new text. However, this method would certainly suffer from data sparseness. Instead, we use a similar approach, but back off from lexical items to parts of speech: we identify as a base NP any string having the same part-of-speech tag sequence as a base NP from the training corpus. The training phase of the algorithm employs two previously successful techniques: like Charniak's (1996) statistical parser, our initial base NP grammar is read from a "treebank" corpus; then the grammar is improved by selecting rules with high "benefit" scores. Our benefit measure is identical to that used in transformation-based learning to select an ordered set of useful transformations (Brill, 1995).

Using this simple algorithm with a naive heuristic for matching rules, we achieve surprising accuracy in an evaluation on two base NP corpora of varying complexity, both derived from the Penn Treebank WSJ. The first base NP corpus is that used in the Ramshaw \& Marcus work. The second espouses a slightly simpler definition of base NP that conforms to the base NPs used in our Empire sentence analyzer. These simpler phrases appear to be a good starting point for partial parsers that purposely delay all complex attachment decisions to later phases of processing.

Overall results for the approach are promising. For the Empire corpus, our base NP finder achieves $94 \%$ precision and recall; for the Ramshaw \& Marcus corpus, it obtains $91 \%$ precision and recall, which is $2 \%$ less than the best published results. Ramshaw \& Marcus, however, provide the learning algorithm with word-level information in addition to the partof-speech information used in our base NP finder. By controlling for this disparity in available knowledge sources, we find that our base NP algorithm performs comparably, achieving slightly worse precision $(-1.1 \%)$ and slightly better recall $(+0.2 \%)$ than the Ramshaw \& Marcus approach. Moreover, our approach offers many important advantages that make it appropriate for many NLP tasks:

- Training is exceedingly simple.

- The base NP bracketer is very fast, operating in time linear in the length of the text.

- The accuracy of the treebank approach is good for applications that require or prefer fairly simple base NPs.

- The learned grammar is easily modified for use with corpora that differ from the training texts. Rules can be selectively added to or deleted from the grammar without worrying about ordering effects.
- Finally, our benefit-based training phase offers a simple, general approach for extracting grammars other than noun phrase grammars from annotated text.

Note also that the treebank approach to base NP identification obtains good results in spite of a very simple algorithm for "parsing" base NPs. This is extremely encouraging, and our evaluation suggests at least two areas for immediate improvement. First, by replacing the naive match heuristic with a probabilistic base NP parser that incorporates lexical preferences, we would expect a nontrivial increase in recall and precision. Second, many of the remaining base NP errors tend to follow simple patterns; these might be corrected using localized, learnable repair rules.

The remainder of the paper describes the specifics of the approach and its evaluation. The next section presents the training and application phases of the treebank approach to base NP identification in more detail. Section 3 describes our general approach for pruning the base NP grammar as well as two instantiations of that approach. The evaluation and a discussion of the results appear in Section 4, along with techniques for reducing training time and an initial investigation into the use of local repair heuristics.

\section{The Treebank Approach}

Figure 2 depicts the treebank approach to base NP identification. For training, the algorithm requires a corpus that has been annotated with base NPs. More specifically, we assume that the training corpus is a sequence of words $w_{1}, w_{2}, \ldots$, along with a set of base NP annotations $b_{\left(i_{1}, j_{1}\right)}, b_{\left(i_{2}, j_{2}\right)}, \ldots$, where $b_{(i, j)}$ indicates that the NP brackets words $i$ through $j$ : [NP $w_{i}, \ldots, w_{j}$ ]. The goal of the training phase is to create a base NP grammar from this training corpus:

1. Using any available part-of-speech tagger, assign a part-of-speech tag $t_{i}$ to each word $w_{i}$ in the training corpus.

2. Extract from each base noun phrase $b_{(i, j)}$ in the training corpus its sequence of part-of-speech tags $t_{i}, \ldots, t_{j}$ to form base NP rules, one rule per base NP.

3. Remove any duplicate rules.

The resulting "grammar" can then be used to identify base NPs in a novel text.

1. Assign part-of-speech tags $t_{1}, t_{2}, \ldots$ to the input words $w_{1}, w_{2}, \ldots$

2. Proceed through the tagged text from left to right, at each point matching the NP rules against the remaining part-of-speech tags $t_{i}, t_{i+1}, \ldots$ in the text. 
Training Phase

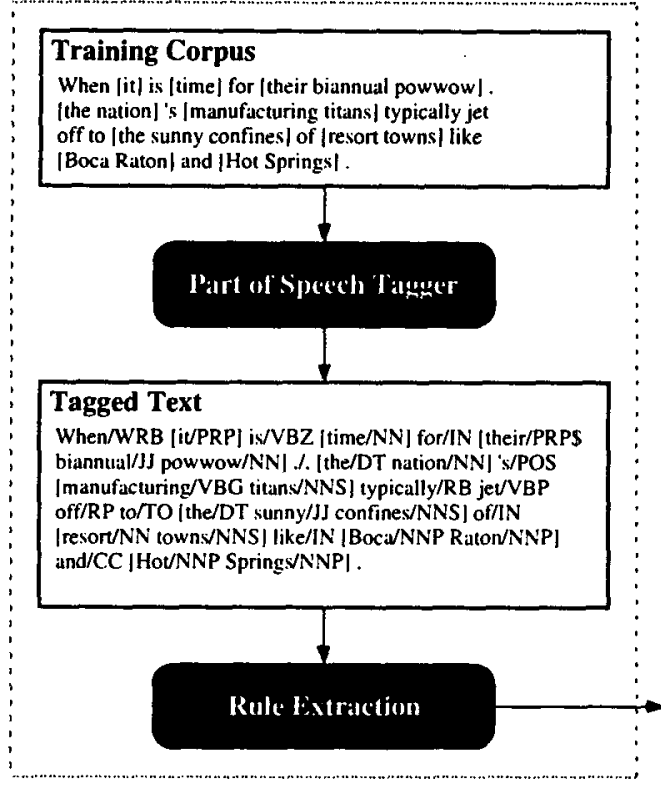

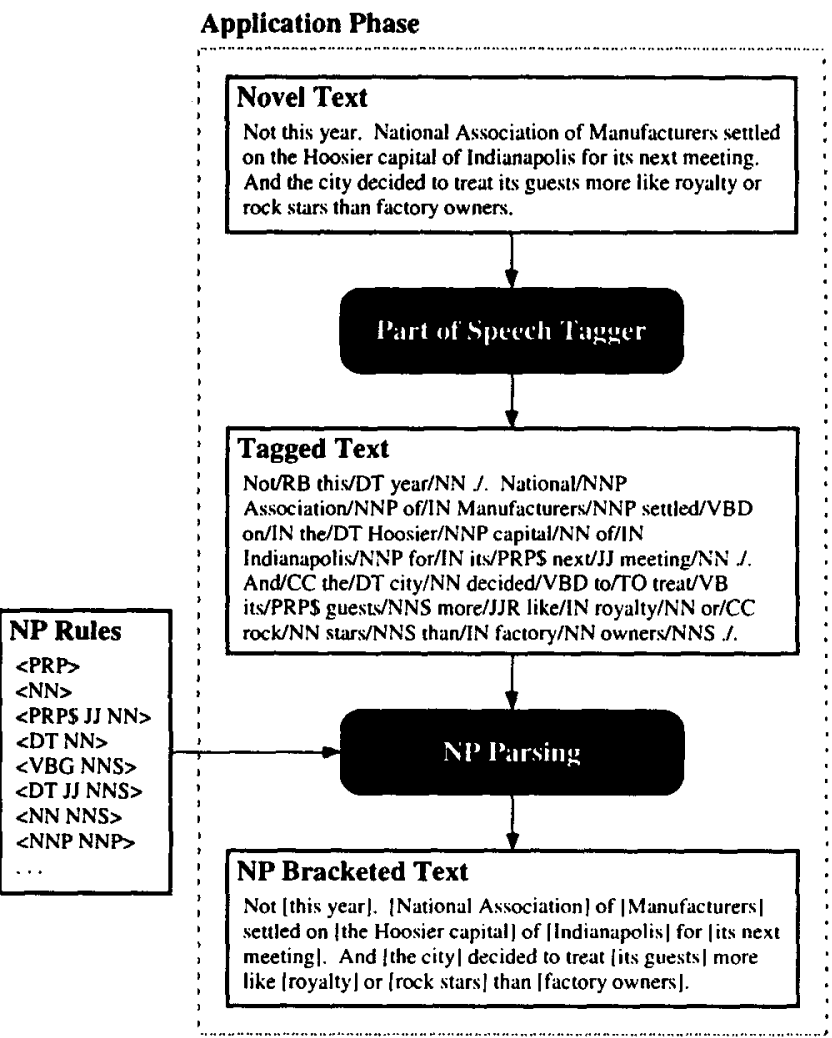

Figure 2: The Treebank Approach to Base NP Identification

3. If there are multiple rules that match beginning at $t_{i}$, use the longest matching rule $R$. Add the new base noun phrase $b_{(i, i+|R|-1)}$ to the set of base NPs. Continue matching at $t_{i+|R|}$.

With the rules stored in an appropriate data structure, this greedy "parsing" of base NPs is very fast. In our implementation, for example, we store the rules in a decision tree, which permits base NP identification in time linear in the length of the tagged input text when using the longest match heuristic.

Unfortunately, there is an obvious problem with the algorithm described above. There will be many unhelpful rules in the rule set extracted from the training corpus. These "bad" rules arise from four sources: bracketing errors in the corpus; tagging errors; unusual or irregular linguistic constructs (such as parenthetical expressions); and inherent ambiguities in the base NPs - in spite of their simplicity. For example, the rule 〈VBG NNS), which was extracted from manufacturing/VBG titans/NNS in the example text, is ambiguous, and will cause erroneous bracketing in sentences such as The execs squeezed in a few meetings before [boarding/VBG buses/NNS] again. In order to have a viable mechanism for identifying base NPs using this algorithm, the grammar must be improved by removing problematic rules.
The next section presents two such methods for automatically pruning the base NP grammar.

\section{Pruning the Base NP Grammar}

As described above, our goal is to use the base NP corpus to extract and select a set of noun phrase rules that can be used to accurately identify base NPs in novel text. Our general pruning procedure is shown in Figure 3. First, we divide the base NP corpus into two parts: a training corpus and a pruning corpus. The initial base NP grammar is extracted from the training corpus as described in Section 2. Next, the pruning corpus is used to evaluate the set of rules and produce a ranking of the rules in terms of their utility in identifying base NPs. More specifically, we use the rule set and the longest match heuristic to find all base NPs in the pruning corpus. Performance of the rule set is measured in terms of labeled precision $(P)$ :

$$
P=\frac{\# \text { of correct proposed NPs }}{\# \text { of proposed NPs }}
$$

We then assign to each rule a score that denotes the "net benefit" achieved by using the rule during NP parsing of the improvement corpus. The benefit of rule $r$ is given by $B_{r}=C_{r}-E_{r}$ where $C_{r}$ 


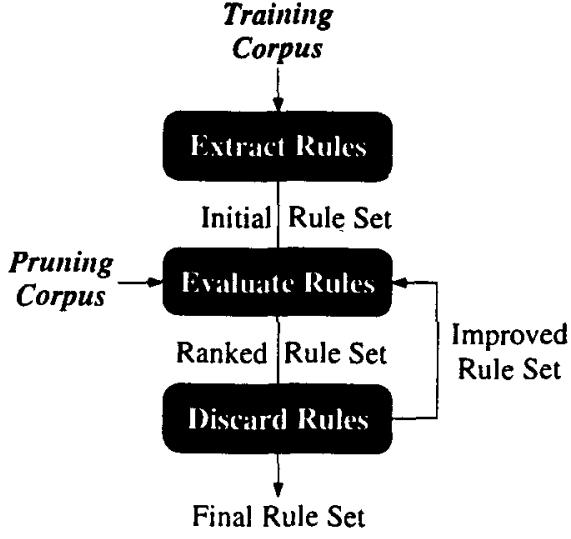

Figure 3: Pruning the Base NP Grammar

is the number of NPs correctly identified by $r$, and $E_{r}$ is the number of precision errors for which $r$ is responsible. ${ }^{1}$ A rule is considered responsible for an error if it was the first rule to bracket part of a reference NP, i.e., an NP in the base NP training corpus. Thus, rules that form erroneous bracketings are not penalized if another rule previously bracketed part of the same reference NP.

For example, suppose the fragment containing base NPs Boca Raton, Hot Springs, and Palm Beach is bracketed as shown below.

resort towns like

$\left[\mathrm{NP}_{1}\right.$ Boca/NNP Raton/NNP , Hot/NNP]

$\left[\mathrm{NP}_{2}\right.$ Springs/NNP], and

$\left[\mathrm{NP}_{3} \mathrm{Palm} / \mathrm{NNP}\right.$ Beach/NNP]

Rule 〈NNP NNP , NNP) brackets $\mathrm{NP}_{1} ;$ (NNP〉 brackets $\mathrm{NP}_{2}$; and $\left\langle\mathrm{NNP} \mathrm{NNP}\right.$ ) brackets $\mathrm{NP}_{3}$. Rule (NNP NNP , NNP) incorrectly identifies Boca Raton, Hot as a noun phrase, so its score is -1 . Rule (NNP) incorrectly identifies Springs, but it is not held responsible for the error because of the previous error by $\langle$ NNP NNP , NNP $\rangle$ on the same original NP Hot Springs: so its score is 0. Finally, rule (NNP NNP) receives a score of 1 for correctly identifying Palm Beach as a base NP.

The benefit scores from evaluation on the pruning corpus are used to rank the rules in the grammar. With such a ranking, we can improve the rule set by discarding the worst rules. Thus far, we have investigated two iterative approaches for discarding rules, a thresholding approach and an incremental approach. We describe each, in turn, in the subsections below.

\footnotetext{
${ }^{1}$ This same benefit measure is also used in the R\&M study, but it is used to rank transformations rather than to rank NP rules.
}

\subsection{Threshold Pruning}

Given a ranking on the rule set, the threshold algorithm simply discards rules whose score is less than a predefined threshold $R$. For all of our experiments, we set $R=1$ to select rules that propose more correct bracketings than incorrect. The process of evaluating, ranking, and discarding rules is repeated until no rules have a score less than $R$. For our evaluation on the WSJ corpus, this typically requires only four to five iterations.

\subsection{Incremental Pruning}

Thresholding provides a very coarse mechanism for pruning the NP grammar. In particular, because of interactions between the rules during bracketing, thresholding discards rules whose score might increase in the absence of other rules that are also being discarded. Consider, for example, the Boca $R a$ ton fragments given earlier. In the absence of $\langle\mathrm{NNP}$ NNP , NNP), the rule (NNP NNP) would have received a score of three for correctly identifying all three NPs.

As a result, we explored a more fine-grained method of discarding rules: Each iteration of incremental pruning discards the $N$ worst rules, rather than all rules whose rank is less than some threshold. In all of our experiments, we set $N=10$. As with thresholding, the process of evaluating, ranking, and discarding rules is repeated, this time until precision of the current rule set on the pruning corpus begins to drop. The rule set that maximized precision becomes the final rule set.

\subsection{Human Review}

In the experiments below, we compare the thresholding and incremental methods for pruning the NP grammar to a rule set that was pruned by hand. When the training corpus is large, exhaustive review of the extracted rules is not practical. This is the case for our initial rule set, culled from the WSJ corpus, which contains approximately 4500 base NP rules. Rather than identifying and discarding individual problematic rules, our reviewer identified problematic classes of rules that could be removed from the grammar automatically. In particular, the goal of the human reviewer was to discard rules that introduced ambiguity or corresponded to overly complex base NPs. Within our partial parsing framework, these NPs are better identified by more informed components of the NLP system. Our reviewer identified the following classes of rules as possibly troublesome: rules that contain a preposition, period, or colon; rules that contain WH tags; rules that begin/end with a verb or adverb; rules that contain pronouns with any other tags; rules that contain misplaced cornmas or quotes; rules that end with adjectives. Rules covered under any of these classes 
were omitted from the human-pruned rule sets used in the experiments of Section 4.

\section{Evaluation}

To evaluate the treebank approach to base NP identification, we created two base NP corpora. Each is derived from the Penn Treebank WSJ. The first corpus attempts to duplicate the base NPs used the Ramshaw \& Marcus (R\&M) study. The second corpus contains slightly less complicated base NPs base NPs that are better suited for use with our sentence analyzer, Empire. ${ }^{2}$ By evaluating on both corpora, we can measure the effect of noun phrase complexity on the treebank approach to base NP identification. In particular, we hypothesize that the treebank approach will be most appropriate when the base NPs are sufficiently simple.

For all experiments, we derived the training, pruning, and testing sets from the 25 sections of Wall Street Journal distributed with the Penn Treebank II. All experiments employ 5 -fold cross validation. More specifically, in each of five runs, a different fold is used for testing the final, pruned rule set; three of the remaining folds comprise the training corpus (to create the initial rule set); and the final partition is the pruning corpus (to prune bad rules from the initial rule set). All results are averages across the five folds. Performance is measured in terms of precision and recall. Precision was described earlier - it is a standard measure of accuracy. Recall, on the other hand, is an attempt to measure coverage:

$$
\begin{aligned}
& P=\frac{\# \text { of correct proposed NPs }}{\# \text { of proposed NPs }} \\
& R=\frac{\# \text { of correct proposed NPs }}{\# \text { of NPs in the annotated text }}
\end{aligned}
$$

Table 1 summarizes the performance of the treebank approach to base NP identification on the $\mathrm{R} \& \mathrm{M}$ and Empire corpora using the initial and pruned rule sets. The first column of results shows the performance of the initial, unpruned base NP grammar. The next two columns show the performance of the automatically pruned rule sets. The final column indicates the performance of rule sets that had been pruned using the handcrafted pruning heuristics. As expected, the initial rule set performs quite poorly. Both automated approaches provide significant increases in both recall and precision. In addition, they outperform the rule set pruned using handcrafted pruning heuristics.

\footnotetext{
${ }^{2}$ Very briefly, the Empire sentence analyzer relies on partial parsing to find simple constituents like base NPs and verb groups. Machine learning algorithms then operate on the output of the partial parser to perform all attachment decisions. The ultimate output of the parser is a semantic case frame representation of the functional structure of the input sentence.
}

\begin{tabular}{|c|c|c|}
\hline $\begin{array}{c}\text { R\&M (1998) } \\
\text { with } \\
\text { lexical templates }\end{array}$ & $\begin{array}{c}\text { R\&M (1998) } \\
\text { without } \\
\text { lexical templates }\end{array}$ & $\begin{array}{c}\text { Treebank } \\
\text { Approach }\end{array}$ \\
\hline \hline $93.1 \mathrm{P} / 93.5 \mathrm{R}$ & $90.5 \mathrm{P} / 90.7 \mathrm{R}$ & $89.4 \mathrm{P} / 90.9 \mathrm{R}$ \\
\hline
\end{tabular}

Table 2: Comparison of Treebank Approach with Ramshaw \& Marcus (1998) both With and Without Lexical Templates, on the R\&M Corpus

Throughout the table, we see the effects of base NP complexity - the base NPs of the R\&M corpus are substantially more difficult for our approach to identify than the simpler NPs of the Empire corpus. For the R\&M corpus, we lag the best published results $(93.1 \mathrm{P} / 93.5 \mathrm{R})$ by approximately $3 \%$. This straightforward comparison, however, is not entirely appropriate. Ramshaw \& Marcus allow their learning algorithm to access word-level information in addition to part-of-speech tags. The treebank approach, on the other hand, makes use only of part-ofspeech tags. Table 2 compares Ramshaw \& Marcus' (In press) results with and without lexical knowledge. The first column reports their performance when using lexical templates; the second when lexical templates are not used; the third again shows the treebank approach using incremental pruning. The treebank approach and the $R \& M$ approach without lecial templates are shown to perform comparably $(-1.1 \mathrm{P} /+0.2 \mathrm{R})$. Lexicalization of our base NP finder will be addressed in Section 4.1.

Finally, note the relatively small difference between the threshold and incremental pruning methods in Table 1. For some applications, this minor drop in performance may be worth the decrease in training time. Another effective technique to speed up training is motivated by Charniak's (1996) observation that the benefit of using rules that only occurred once in training is marginal. By discarding these rules before pruning, we reduce the size of the initial grammar - and the time for incremental pruning - by $60 \%$, with a performance drop of only $-0.3 P /-0.1 R$.

\subsection{Errors and Local Repair Heuristics}

It is informative to consider the kinds of errors made by the treebank approach to bracketing. In particular, the errors may indicate options for incorporating lexical information into the base NP finder. Given the increases in performance achieved by Ramshaw \& Marcus by including word-level cues, we would hope to see similar improvements by exploiting lexical information in the treebank approach. For each corpus we examined the first 100 or so errors and found that certain linguistic constructs consistently cause trouble. (In the examples that follow, the bracketing shown is the error.) 


\begin{tabular}{|c||c|c|c|c|}
\hline $\begin{array}{c}\text { Base NP } \\
\text { Corpus }\end{array}$ & $\begin{array}{c}\text { Initial } \\
\text { Rule Set }\end{array}$ & $\begin{array}{c}\text { Threshold } \\
\text { Pruning }\end{array}$ & $\begin{array}{c}\text { Incremental } \\
\text { Pruning }\end{array}$ & $\begin{array}{c}\text { Human } \\
\text { Review }\end{array}$ \\
\hline \hline Empire & $23.0 \mathrm{P} / 46.5 \mathrm{R}$ & $91.2 \mathrm{P} / 93.1 \mathrm{R}$ & $92.7 \mathrm{P} / 93.7 \mathrm{R}$ & $90.3 \mathrm{P} / 90.5 \mathrm{R}$ \\
\hline $\mathrm{R} \& \mathrm{M}$ & $19.0 \mathrm{P} / 36.1 \mathrm{R}$ & $\mathbf{8 7 . 2 \mathrm { P } / 9 0 . 0 \mathrm { R }}$ & $89.4 \mathrm{P} / 90.9 \mathrm{R}$ & $81.6 \mathrm{P} / 85.0 \mathrm{R}$ \\
\hline
\end{tabular}

Table 1: Evaluation of the Treebank Approach Using the Mitre Part-of-Speech Tagger $(P=$ precision; $R=$ recall)

\begin{tabular}{|c||c|c||c|c|}
\hline $\begin{array}{c}\text { Base NP } \\
\text { Corpus }\end{array}$ & $\begin{array}{c}\text { Threshold } \\
\text { Improvement }\end{array}$ & $\begin{array}{c}\text { Threshold } \\
+ \text { Local Repair }\end{array}$ & $\begin{array}{c}\text { Incremental } \\
\text { Improvement }\end{array}$ & $\begin{array}{c}\text { Incremental } \\
\text { + Local Repair }\end{array}$ \\
\hline \hline Empire & $91.2 \mathrm{P} / \overline{9} .1 \mathrm{R}$ & $92.8 \mathrm{P} / 93.7 \mathrm{R}$ & $92.7 \mathrm{P} / 93.7 \mathrm{R}$ & $93.7 \mathrm{P} / 94.0 \mathrm{R}$ \\
\hline$\overline{\mathrm{R} \& \mathrm{M}}$ & $87.2 \mathrm{P} / 90.0 \mathrm{R}$ & $89.2 \mathrm{P} / 90.6 \mathrm{R}$ & $89.4 \mathrm{P} / 90.9 \mathrm{R}$ & $90.7 \mathrm{P} / 91.1 \mathrm{R}$ \\
\hline
\end{tabular}

Table 3: Effect of Local Repair Heuristics

- Conjunctions. Conjunctions were a major problem in the R\&M corpus. For the Empire corpus, conjunctions of adjectives proved difficult: $[$ record/NM $[$ third-quarter $/ J J$ and $/ C C$ nine-month/JJ results/NNS].

- Gerunds. Even though the most difficult VBG constructions such as manufacturing titans were removed from the Empire corpus, there were others that the bracketer did not handle, like [chief] operating [officer]. Like conjunctions, gerunds posed a major difficulty in the R\&M corpus.

- NPs Containing Punctuation. Predictably, the bracketer has difficulty with NPs containing periods, quotation marks, hyphens, and parentheses.

- Adverbial Noun Phrases. Especially temporal NPs such as last month in at [83.6\%] of [capacity last month].

- Appositives. These are juxtaposed NPs such as of [colleague Michael Madden] that the bracketer mistakes for a single NP.

- Quantified NPs. NPs that look like PPs are a problem: at/IN [least/JJS] [the/DT right/JJ jobs/NNS]; about/IN [25/CD million/CD].

Many errors appear to stem from four underlying causes. First, close to $20 \%$ can be attributed to errors in the Treebank and in the Base NP corpus, bringing the effective performance of the algorithm to $94.2 \mathrm{P} / 95.9 \mathrm{R}$ and $91.5 \mathrm{P} / 92.7 \mathrm{R}$ for the $\mathrm{Em}$ pire and R\&M corpora, respectively. For example, neither corpus includes WH-phrases as base NPs. When the bracketer correctly recognizes these NPs, they are counted as errors. Part-of-speech tagging errors are a second cause. Third, many NPs are missed by the bracketer because it lacks the appropriate rule. For example, household products business is bracketed as [household/NN products/NNS]
[business/NN]. Fourth, idiomatic and specialized expressions, especially time, date, money, and numeric phrases, also account for a substantial portion of the errors.

These last two categories of errors can often be detected because they produce either recognizable patterns or unlikely linguistic constructs. Consecutive NPs, for example, usually denote bracketing errors, as in [household/NN products/NNS] [business/NM]. Merging consecutive NPs in the correct contexts would fix many such errors. Idiomatic and specialized expressions might be corrected by similarly local repair heuristics. Typical examples might include changing [effective/JJ Monday/NNP] to effective [Monday]; changing [the/DT balance/NN due/JJ] to [the balance] due; and changing were/VBP [n't/RB the $/ D T$ only/RB losers/NNS] to were n't [the only losers].

Given these observations, we implemented three local repair heuristics. The first merges consecutive NPs unless either might be a time expression. The second identifies two simple date expressions. The third looks for quantifiers preceding of $N P$. The first heuristic, for example, merges [household products] [business] to form [household products business], but leaves increased [15\%] [last Friday] untouched. The second heuristic merges [June 5], [1995] into [June $5,1995]$; and [June] , [1995] into [June, 1995]. The third finds examples like some of [the companies] and produces [some] of [the companies]. These heuristics represent an initial exploration into the effectiveness of employing lexical information in a post-processing phase rather than during grammar induction and bracketing. While we are investigating the latter in current work, local repair heuristics have the advantage of keeping the training and bracketing algorithms both simple and fast.

The effect of these heuristics on recall and precision is shown in Table 3 . We see consistent improvements for both corpora and both pruning methods, 
achieving approximately $94 \mathrm{P} / \mathrm{R}$ for the Empire corpus and approximately $91 \mathrm{P} / \mathrm{R}$ for the $\mathrm{R} \& \mathrm{M}$ corpus. Note that these are the final results reported in the introduction and conclusion. Although these experiments represent only an initial investigation into the usefulness of local repair heuristics, we are very encouraged by the results. The heuristics uniformly boost precision without harming recall; they help the R\&M corpus even though they were designed in response to errors in the Empire corpus. In addition, these three heuristics alone recover $1 / 2$ to $1 / 3$ of the improvements we can expect to obtain from lexicalization based on the $R \& M$ results.

\section{Conclusions}

This paper presented a new method for identifying base NPs. Our treebank approach uses the simple technique of matching part-of-speech tag sequences, with the intention of capturing the simplicity of the corresponding syntactic structure. It employs two existing corpus-based techniques: the initial noun phrase grammar is extracted directly from an annotated corpus; and a benefit score calculated from errors on an improvement corpus selects the best subset of rules via a coarse- or fine-grained pruning algorithm.

The overall results are surprisingly good, especially considering the simplicity of the method. It achieves $94 \%$ precision and recall on simple base NPs. It achieves $91 \%$ precision and recall on the more complex NPs of the Ramshaw \& Marcus corpus. We believe, however, that the base NP finder can be improved further. First, the longest-match heuristic of the noun phrase bracketer could be replaced by more sophisticated parsing methods that account for lexical preferences. Rule application, for example, could be disambiguated statistically using distributions induced during training. We are currently investigating such extensions. One approach closely related to ours - weighted finite-state transducers (e.g. (Pereira and Riley, 1997)) - might provide a principled way to do this. We could then consider applying our error-driven pruning strategy to rules encoded as transducers. Second, we have only recently begun to explore the use of local repair heuristics. While initial results are promising, the full impact of such heuristics on overall performance can be determined only if they are systematically learned and tested using available training data. Future work will concentrate on the corpusbased acquisition of local repair heuristics.

In conclusion, the treebank approach to base NPs provides an accurate and fast bracketing method, running in time linear in the length of the tagged text. The approach is simple to understand, implement, and train. The learned grammar is easily modified for use with new corpora, as rules can be added or deleted with minimal interaction problems. Finally, the approach provides a general framework for developing other treebank grammars (e.g., for subject/verb/object identification) in addition to these for base NPs.

Acknowledgments. This work was supported in part by NSF Grants IRI-9624639 and GER-9454149. We thank Mitre for providing their part-of-speech tagger.

\section{References}

D. Bourigault. 1992. Surface Grammatical Analysis for the Extraction of Terminological Noun Phrases. In Proceedings, COLING-92, pages 977981.

Eric Brill. 1995. Transformation-Based ErrorDriven Learning and Natural Language Processing: A Case Study in Part-of-Speech Tagging. Computational Linguistics, 21(4):543-565.

E. Charniak. 1996. Treebank Grammars. In Proceedings of the Thirteenth National Conference on Artificial Intelligence, pages 1031-1036, Portland, OR. AAAI Press / MIT Press.

K. Church. 1988. A Stochastic Parts Program and Noun Phrase Parser for Unrestricted Text. In Proceedings of the Second Conference on Applied Natural Language Processing, pages 136-143. Association for Computational Linguistics.

J. P. Gee and F. Grosjean. 1983. Performance structures: A psycholinguistic and linguistic appraisal. Cognitive Psychology, 15:411-458.

John S. Justeson and Slava M. Katz. 1995. Technical Terminology: Some Linguistic Properties and an Algorithm for Identification in Text. Natural Language Engineering, 1:9-27.

M. Marcus, M. Marcinkiewicz, and B. Santorini. 1993. Building a Large Annotated Corpus of English: The Penn Treebank. Computational Linguistics, 19(2):313-330.

Fernando C. N. Pereira and Michael D. Riley. 1997. Speech Recognition by Composition of Weighted Finite Automata. In Emmanuel Roche and Yves Schabes, editors, Finite-State Language Processing. MIT Press.

Lance A. Ramshaw and Mitchell P. Marcus. In press. Text chunking using transformation-based learning. In Natural Language Processing Using Very Large Corpora. Kluwer. Originally appeared in WVLC95, 82-94.

A. Voutilainen. 1993. NPTool, A Detector of English Noun Phrases. In Proceedings of the Workshop on Very Large Corpora, pages 48-57. Association for Computational Linguistics. 